\title{
Preface
}

\section{Advances in Screening and Prevention of Women's Cancers}

\section{Editor- Prof Ranjit Manchanda}

GLOBOCAN suggests the burden of cancers is set to increase by $57 \%$ over the next 20 years. The number of cases of breast, ovarian, endometrial and cervical cancers worldwide is expected to rise by $46.5 \%, 47 \%, 53 \%$ and $36 \%$ respectively.[1] Correspondingly the number of deaths from these cancers worldwide is predicted to increase by $58.3 \%, 58.6 \%, 71 \%$ and $48 \%$ respectively by 2040 .[1] Chronic disease of which cancer is the second commonest cause is the greatest challenge facing our health systems. It accounts for $90 \%$ Medicare[2] and 70\% UK health care expenditure.[3] Arguably, the largest impacts on the burden of disease have historically come through preventive interventions. However, most research and health funding remains focused on treatment and diagnosis of disease rather than screening and prevention. There is a need to re-balance resource allocation to address this inequity. Chronic disease prevention could reduce treatment costs and increase GDP by \$billions[4]. Improving screening and prevention is critical for the future financial sustainability of our health systems. There is emerging recognition of this, with policy makers now highlighting the increasing importance of this in some countries.[5]

This issue of Best Practice and Research in Clinical Obstetrics and Gynaecology, provides an update on some of the advances in screening and prevention of Women's Cancers. While, a huge amount of work remains to be done, there have been a number of strides and achievements over the last decade or so across the field of screening and prevention of women's cancers. Harkness et al, provide an overview of risk adapted breast cancer screening, a new emerging paradigm in screening women for breast cancer. A number of effective preventive strategies are available for women at high risk of breast cancer and Thorat et al, provide an update on this field. Screening for ovarian cancer remains a huge challenge. There have been a number of ovarian cancer screening trials in the low and high-risk populations. While a stage shift has been demonstrated, a conclusive mortality impact has not. No country currently offers a national ovarian cancer screening programme. Nash et al, provide an overview of the current state of play and potential future direction of travel. Surgical prevention of ovarian cancer is both clinically effective and cost-effective. There have been calls for broadening access for women at increased risk. Additionally, salpingectomy strategies are emerging as options for low and high-risk women. Premenopausal oophorectomy leads to premature surgical menopause with detrimental long-term health consequences. In this issue, Gaba et al, provide a systematic review on acceptability, cardiovascular, neurological, bone health, and HRT outcomes of risk reducing salpingo-oophorectomy in high-risk women. The existence of effective screening and now preventive mechanisms for cervical cancer is one of the success stories of cancer prevention. However, it is still associated with a significant disease burden particularly in low and middle income countries. There is now broad acceptance and a big move towards primary HPV screening for cervical cancer. Bhatla et al, provide a review of primary HPV screening for cervical cancer and Athanasiou et al, cover the role of HPV vaccination for primary prevention. The incidence of endometrial cancer has been rising over the years and it is now the commonest gynaecological malignancy in the Western world. Changing lifestyles and obesity have played a major role in this. There is currently no acceptable screening strategy or programme for endometrial cancer. Njoku et al, summarise the various risk factors and potential prevention strategies for endometrial cancer, and Gentry-Maharaj et al, provide an overview of the screening landscape. Improving ascertainment of women at high risk is key for bettering targeted screening and prevention. This in turn will reduce 
future cancer incidence and mortality. The growing strategy of unselected genetic testing at cancer diagnosis and a novel paradigm of population-based genetic testing in unaffected women (and men) provides the opportunity to reduce burden of disease in a big way. Amin et al, review unselected genetic testing in women with epithelial ovarian cancer and Evans et al, summarise the current evidence on population-based genetic testing for precision prevention.

I am grateful to Prof Arulkumaran and Ms Beverly Burns for their support and to all the authors for their help and efforts in pulling this issue together.

\section{References}

[1] International Agency for Research on Cancer. Cancer Tomorrow. A tool that predicts the future cancer incidence and mortality burden worldwide from the current estimates in 2018 up until 2040. Lyon, France: International Agency for Research on Cancer (IARC); 2018.

[2] Murphy SL, Xu JQ, Kochanek KD, Curtin SC, Arias E. Deaths: Final Data for 2015. National Vital Statistics Reports. 2017;66:https://www.cdc.gov/nchs/data/nvsr/nvsr66/nvsr 06.pdf.

[3] Department of Health Long Term Conditions Team. Long Term Conditions Compendium of Information. Third Edition ed. Leeds: Department of Health; 2012. p.

https://www.gov.uk/government/uploads/system/uploads/attachment data/file/216528/dh 13448 6.pdf.

[4] Milken Institute. Checkup Time: Chronic Disease and Wellness in America. 2014.

[5] NHS England. The NHS Long Term Plan. London, UK: National Health Service, England; 2019. p. https://www.longtermplan.nhs.uk/. 